\title{
Predicting Breast Cancer Incidence Rates Among White and Black Women in the United States: An Application of FTS Model
}

\author{
Farah Yasmeen \\ Department of Statistics, University of Karachi, Karachi, Pakistan
}

Email address:

drfarahyas@gmail.com

\section{To cite this article:}

Farah Yasmeen. Predicting Breast Cancer Incidence Rates Among White and Black Women in the United States: An Application of FTS Model. International Journal of Statistical Distributions and Applications. Vol. 3, No. 4, 2017, pp. 103-112.

doi: $10.11648 /$ j.ijsd.20170304.17

Received: March 10, 2017; Accepted: March 29, 2017; Published: November 28, 2017

\begin{abstract}
Development of statistical model for cancer incidence trend predictions can provide a sound and accurate foundation for planning a comprehensive national strategy for optimal partitioning of research resources. Several studies in the past showed that that there are racial/ethnic disparities exist between breast cancer incidence rates among black and white women in the United States. Some of the studies also showed that the disparity in breast cancer incidence rates among white and black US women is widening, with relatively higher incidence rates among black women. In this paper, we apply functional time series (FTS) models on the age-specific breast cancer incidence rates for these two major groups of women in US, and forecast their age-incidence curves. The data are obtained from the Surveillance, Epidemiology and End Results (SEER) program of the United States. We use annual unadjusted breast cancer incidence rates from 1973 to 2013 in 5 -year agegroups $(15-19,20-24,25-29,30-34,35-39,40-44,45-49,50-54,55-59,60-64,65-69,70-74,75-79$, 80-84 and 85+). Age-specific cancer incidence curves are obtained using nonparametric smoothing methods. The curves are then decomposed using functional data paradigm and we fit functional time series (FTS) models for each population of women separately. The smoothed incidence curves are then forecasted and prediction intervals are calculated. Fifteen-year forecasts indicate an overall increase in future breast cancer incidence rates for both groups of women. This increase appears to be faster among black women and relatively slower among the whites. The projections suggest a need for equal delivery of quality care to eliminate breast cancer disparities among the two major groups of women in US.
\end{abstract}

Keywords: Breast Cancer, Cancer Incidence, Screening and Early Detection, Functional Time Series, Forecasts, Black and White Disparity

\section{Introduction}

Breast cancer is the most common cancer among women worldwide. It is documented that the global burden of breast cancer is expected to increase up to 2 million and over the new cancer cases per year by 2030 [1]. For women in the United States, breast cancer is the most common cancer, excluding skin cancers. It is estimated that there are more than 3.5 million women living in the United States with a history of invasive breast cancer [2]. Several epidemiologic studies suggest that substantial disparities exist between black and white women in their breast cancer incidence. In addition to these disparities, there are substantial black-white differences in other factors, e.g. cancer stage at the time of diagnosis and five-year survival after diagnosis. It is found that compared to white women, black women have an increased risk for stages III and stage-IV tumors, age at diagnosis and year of diagnosis [3-4].Some studies suggested that the black women in comparison with white women are more likely to be diagnosed with breast cancer at a distant stage of the disease [5].

In this paper, we apply the functional time series models of [6] to the age-specific breast cancer incidence rates of white and black women in the United States. These models are previously applied to the breast cancer mortality rates in Australia [7], to the French mortality and Australian fertility rates [6], to the breast cancer mortality among black and 
white women in the United States [8-9] and to the breast cancer incidence rates in Karachi [10-12]. The performance of these models with the most common age-period-cohort models of modeling mortality is compared in [13].

The main objectives of this paper are as follow:

(a) To apply the functional time series (FTS) model, independently to the breast cancer incidence rates of black and white women in the United States.

(b) To study and compare various age and time components FTS Models applied on black and whites incidence data

(c) To obtain 15-year forecasts of age-incidence curves for the two major groups of women in US

(d) To discuss the results obtained from statistical analysis, and to compare them with the results/findings of other studies

This paper is divided into six sections. Section 1 is introductory, while the cancer incidence rates and information about the data source are described in section 2. Some basic concepts of functional time series (FTS) are explained in section 3. Statistical analysis on age-specific breast cancer incidence rates of black and white females is performed in section 4 and the results are discussed and compared with other studies in section 5. Finally, concluding remarks are given in section 5.

\section{Statistical Data of Breast Cancer Incidence in the United States}

Since 1973, the population-based cancer incidence data in the United States have been collected by the National Cancer Institute's Surveillance, Epidemiology, and End Results (SEER) Program [14]. Since 1995, the incidence data are also collected by the Centers for Disease Control and Prevention's National Program of Cancer Registries (NPCR). The rates are available for white, black, American Indians/Alaska Natives and others.

The original SEER data are available for 19 age groups $(<1$ year, 1-4 years, 5-9 years, ..., 85+ years) . However in this study, we analyzed the data for fifteen age groups: 15-19, 20-24, 25-29, $30-34,35-39,40-44,45-49,50-54,55-59,60-64,65-69,70$ $74,75-79,80-84$ and $85+$.The newly diagnosed cases and incidence rates of breast cancer among these fifteen age-groups are investigated, as the incidence rates among the other four groups ( $<1$ year, 1-4 years, 5-9 years and 10-14 years) are extremely small or negligible. All statistical analyses were performed in $\mathrm{R}$ version 3.3.1 using the R packages demography [15] and forecast [16], available at CRAN.

\section{Statistical Methodology}

The Functional Time series (FTS) models are first developed by [6], where they applied them to the Australian fertility and French mortality data. These models werealso applied to the breast cancer mortality in Australia [7] and incidence rates by [9-11]. [12] made an attempt to compare various components of FTS models (in the form of age and time) by applying them independently to the breast cancer incidence data of Karachi (Pakistan) and US (White females). Here, we are going to apply these models to the age-specific incidence rates of breast cancer among black and white women in the United States.

\subsection{The Functional Time Series (FTS) Model}

Suppose $y_{t, j}(x)$ denotes the log incidence rate at age $x$ in year $t$ forthe jth group $(j=1,2)$. Here, white and black women are consideredto be group 1 and 2, respectively. We assume that $s_{t, j}(x)$ is a smooth function of age with

$$
y_{t, j}(x)=s_{t, j}(x)+\sigma_{t, j}(x) \epsilon_{t, j, x}
$$

Where $\sigma_{t, j}(x)$ allows the amountof noise to vary with age $\mathrm{x}$, and $\epsilon_{t, j, x}$ are considered to be independentand identically distributed random variables, having zero mean andunit variance. The first step is to estimate these smooth functions from the discrete noisy data of incidence rates. For this, nonparametric smoothing methods are used such as penalized regression splines or loess curves. The smoothed curves are then decomposed via a basis function expansion using the following model, as described in [6]

$$
s_{t, j}(x)=\mu_{j}(x)+\sum_{k=1}^{K} \beta_{j, k} \emptyset_{j, k}(x)+\varepsilon_{j, t}(x)
$$

Here $\mu_{j}(x)$ is the mean log incidence rate across years for the jth group and $\emptyset_{j, k}(x)$ is a set of orthogonal basis functions for each $\mathrm{j}$. The scores $\beta_{j, k}$ form a univariate time series for each $(j=1,2)$ and $k=1,2, \ldots ., K$. The basis functions $\emptyset_{j, k}(x)$ are computedusing functional principal components applied to the smooth curves $s_{t, j}(x)$. The h-step ahead forecast of $y_{t, j}(x)$ can be obtained as

$$
\hat{y}_{t, j}(x)=\hat{\mu}_{j}(x)+\sum_{k=1}^{K} \hat{\beta}_{j, k} \widehat{\emptyset}_{j, k}(x)
$$

where $\hat{\mu}_{j}(x)$ and $\widehat{\emptyset}_{j, k}(x)$ are the estimates of the mean function and basis functions, respectively and $\hat{\beta}_{j, k}$ denotes the h-step ahead forecast of $\beta_{j, k}$ computed using ARIMA models with automatic forecasting algorithm.[6] also showed that the forecast variance can be obtained by adding the variances of all individual terms as

$$
\sigma_{j, n+h}^{2}(x)
$$

where $\hat{\sigma}_{j, \mu}^{2}(x)$ is the variance obtained using the smoothing method, $v_{j, k, n}$ is the forecast variance of coefficients, obtained from the time series model, $u_{j}(x)$ is obtained using the sum of squared residuals, and $\sigma_{j, n+h}^{2}(x)$ can be obtained using a Poisson approximation (The reader is referred to [6] for details). Assuming a normal distribution for the forecast errors and after substituting the values of all these terms in (4), one may obtain prediction intervals for the entire log incidence curve.

\subsection{Statistical Analysis and Results}

To see the age-related changes in the breast cancer, the 
first step is to plot the age-specific incidence rates. Figure 1 represents the observed breast cancer incidence rates (per 100,000 women) among white and black women in the United States during 1973-2013 for various age-groups. To plot this figure, we use rainbow plot [17] for representing different age-groups. The curves are plotted in a rainbow order with the earlier age group (15-19) is represented by red and the last age-group $(85+)$ is plotted by violet color. The other age groups are appeared in the rainbow order, with Rpackage 'rainbow' is used to construct this plot.

From Figure 1, we can say that the breast cancer incidence rates among white and black US women were increasing with age monotonically, with relatively smaller rates for women under 40 years of age and higher for the women ages 40 years and above. The rates were increasing since 1973 till 1999 and increased very sharply from 1999 to 2000 for all age-groups for both whites and blacks.

The patterns for whites are already discussed in [12]. The breast cancer incidence rates among black women increased during 1973-1999, and this increase is quite sharp from 1999 to 2000 for all age-groups. However, it can be noted from Figure 1 and Figure 2, that the incidence rates for all age groups among black women remained relatively smaller from 1973 till 1999, as compared to the rates among their white counterpart,. Then, the rates for both races increased and became higher for the blacks as compared to the whites.

In order to analyze the incidence rates and to describe the changes in incidence rates with age, we also plot the incidence curves (as function of age) for the years 1973-2013 in Figure 2. The observed log incidence rates and smooth curves of these rates as functional time series observations are plotted in Figure 3. From these figures, we can see that the breast cancer incidence rates increased with age in general. From early ages (around 15), the overall incidence rates are showing a clear increasing trend through age of 50. The rates declined for the white women of age $50+$ in most recent years, whereas the graph shows continuously increasing rates for the black women of age 50 years and older.

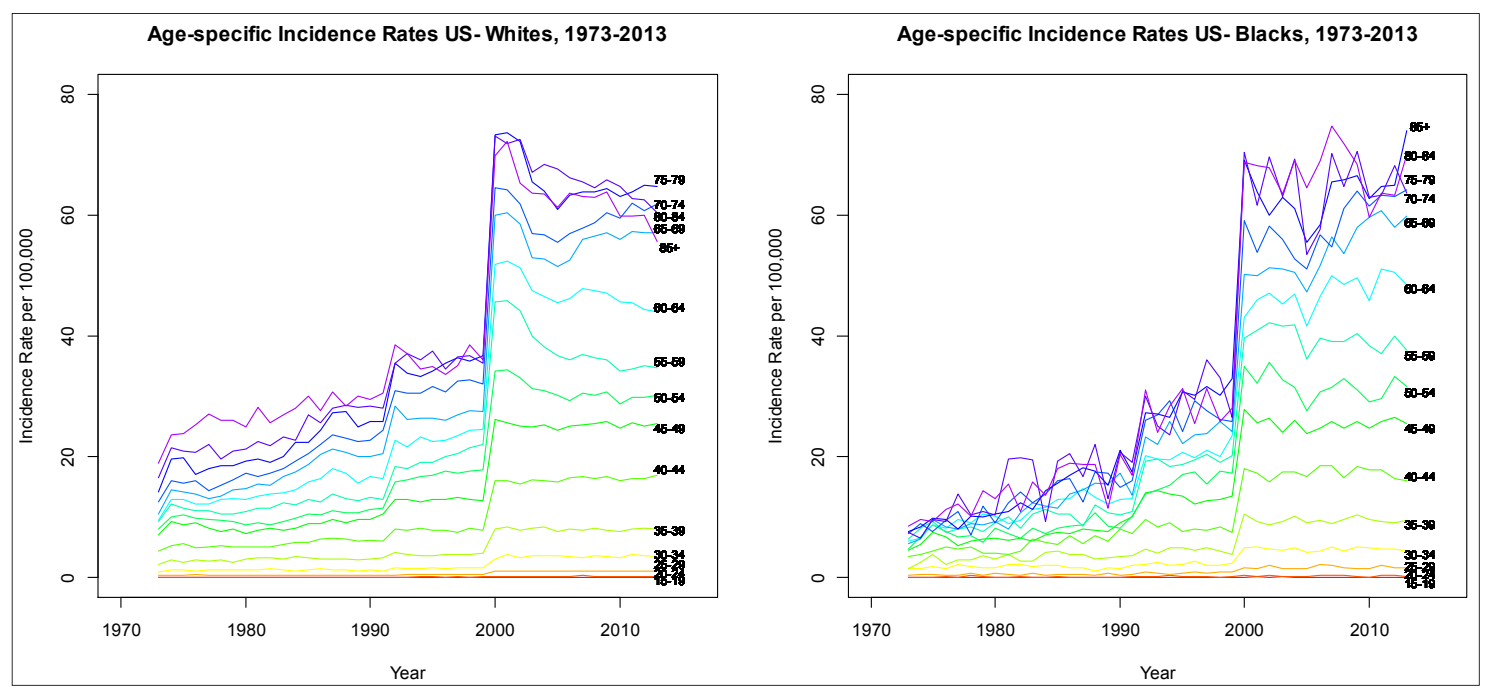

Figure 1. Age-specific breast cancer incidence rates for US-White and US-Black women during (1973-2013) for the age-groups 15-19, 20-24, 25-29, 30-34, $35-39,40-44,45-49,50-54,55-59,60-64,65-69,70-74,75-79,80-84$ and $85+$.
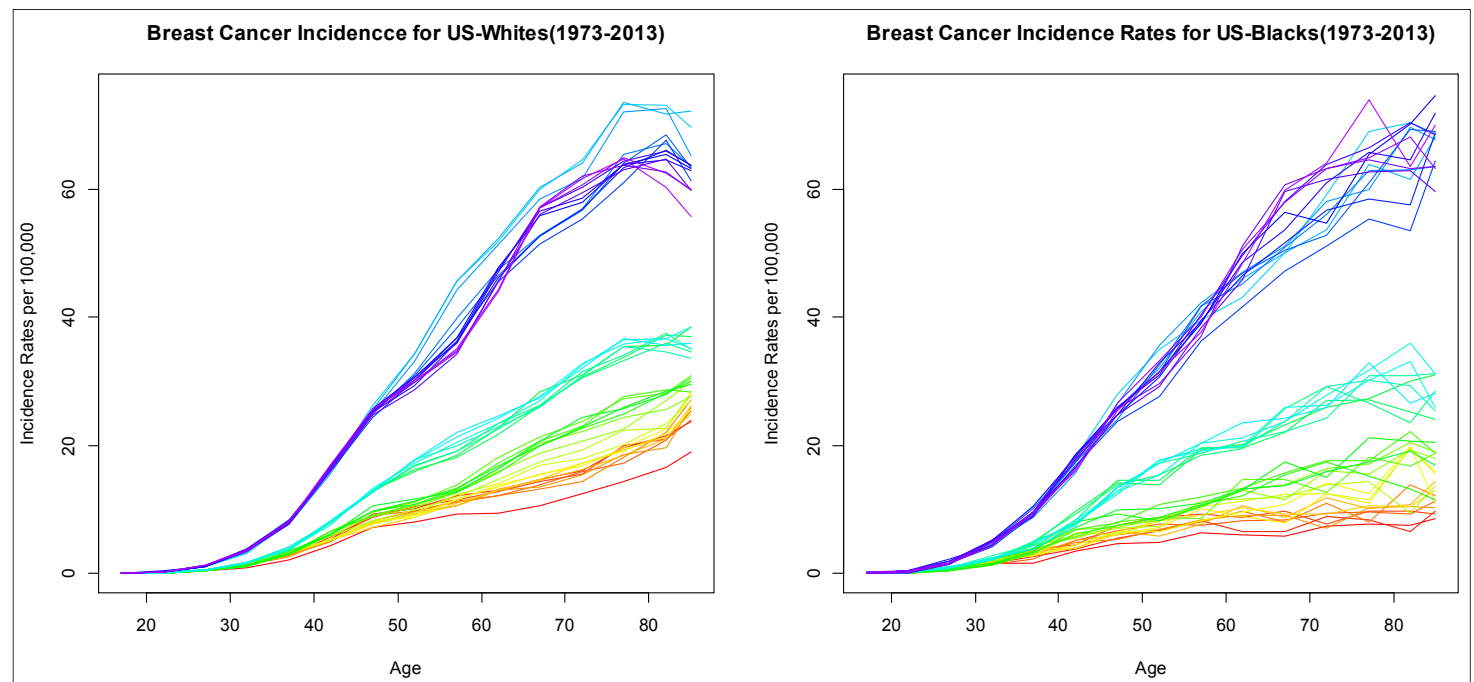

Figure 2. Breast Cancer incidence curves (as function of age) of US-White and US-Black women during 1973-2013. The years are represented in rainbow order, using the rainbow plot [17]. The earlier years are in red, whereas the most recent years are represented by violet colour. 
Next step is to apply functional time series (FTS) model given in equation (2) of [9] to the smoothed log incidence rates (shown in Figure 3). We use a model with 4 basis functions, and the age and time components and their forecasts are depicted in Figure 4 for blacks. The percentage variation due to the first four basis functions is $88.7 \% 10.5 \%$ $0.6 \% 0.2 \%$. It can be noted that the percentage variation for the first component is little bit lower among black women (88.7\%) as compared to whites $(91.3 \%)$ due to more spread (or variation) among the incidence rates of blacks.
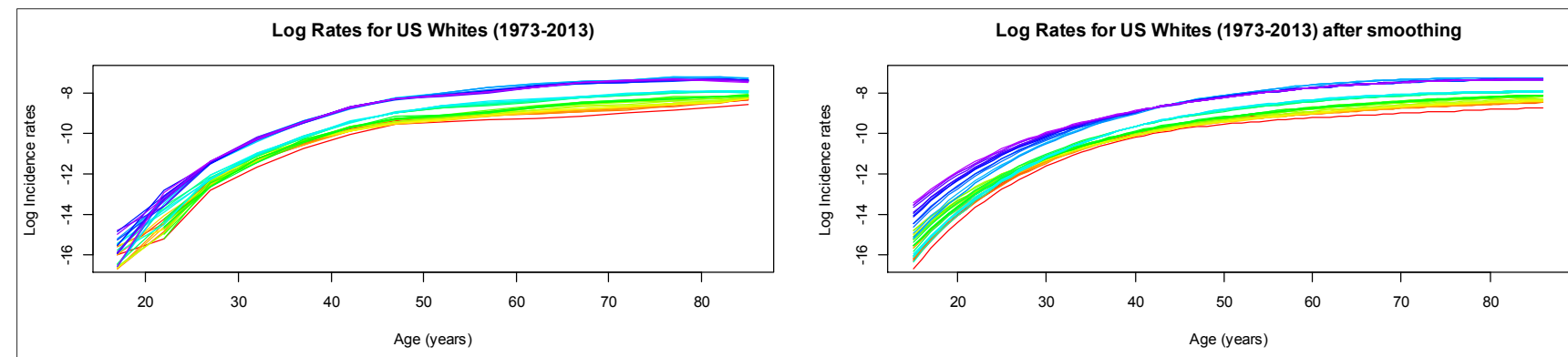

Log Rates for US Blacks(1973-2013)
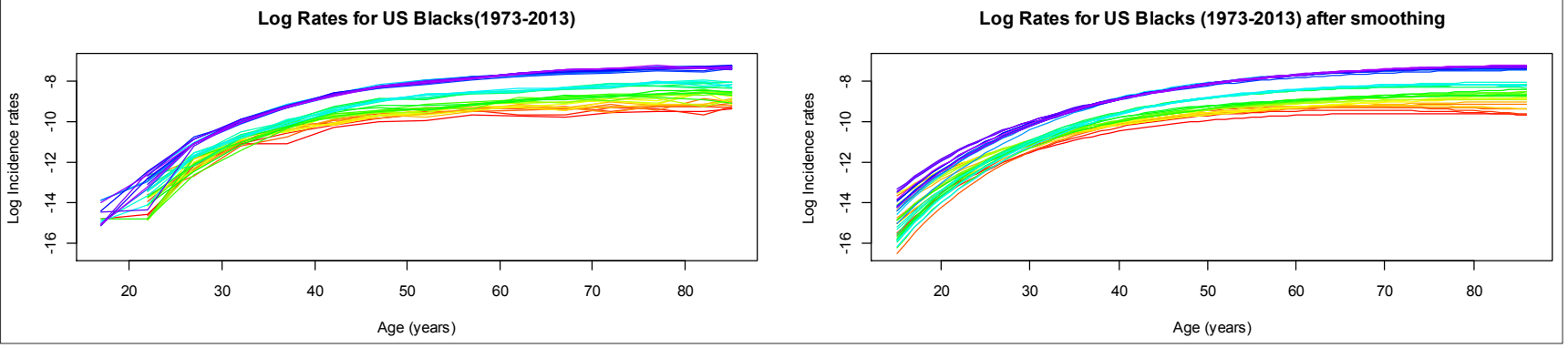

Figure 3. Log incidence rates for US-Whites and US-Black women during (1973-2013) as functional observations. On the left panel, original log incidence rates are plotted whereas the rates after smoothing are plotted on the right panel. Penalized regression splines are used to smooth the log incidence rates.
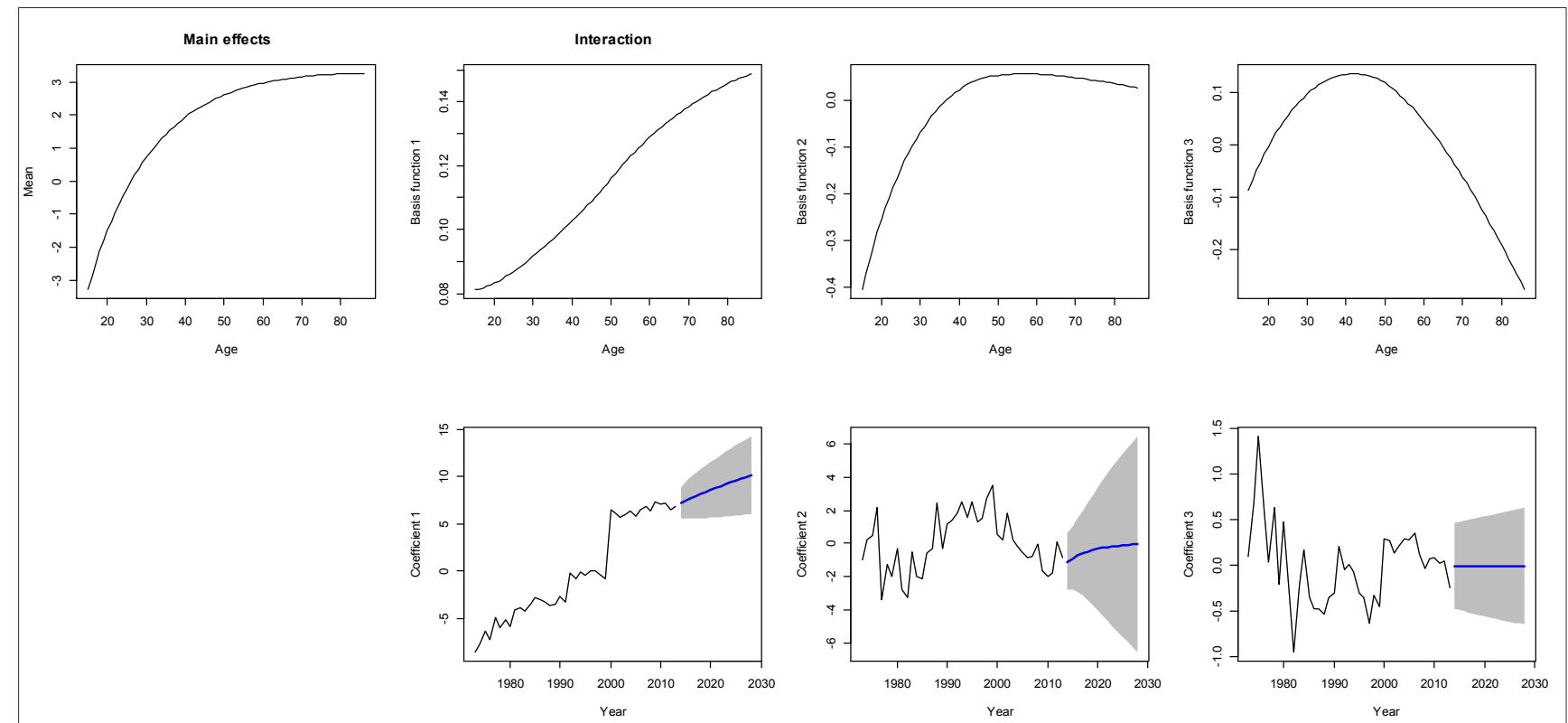

Figure 4. Different components of FTS models applied on Breast Cancer Incidence Rates in US-Black women. The time series coefficients are also plotted along with 15-years forecasts (2014-2028), represented by grey shaded region.

In Figure 4, the mean function for black women shows a similar pattern as for the whites, i.e increase in the breast cancer incidence rates with age, however; the other components of FTS models are all different. The first basis function for blacks represents those US black women who are very old (about 50 years and above). The respective time series coefficient shows that the incidence rates of those black women were increasing since 1973, increased very sharply from 1999 to 2000, and then increased relatively slowly till 2013. 15-year forecast of this time series coefficient shows that future incidence rates are expected to increase for older black females (above 50-years). 
The second basis function from Figure 4 again represents older black women (40 years and above) and corresponding time series coefficient shows that the incidence rates among these women were increased since 1973 to 2000, then decreased slightly from 2000 to 2013 . The future incidence rates in this age group are expected to increase in the next 15- years. The other components of FTS model are not interpreted. We have plotted the first basis function and the first coefficients of whites and blacks separately in Figure 5 and Figure 6 again, to show the difference among the two basis functions and similarity among their coefficients.

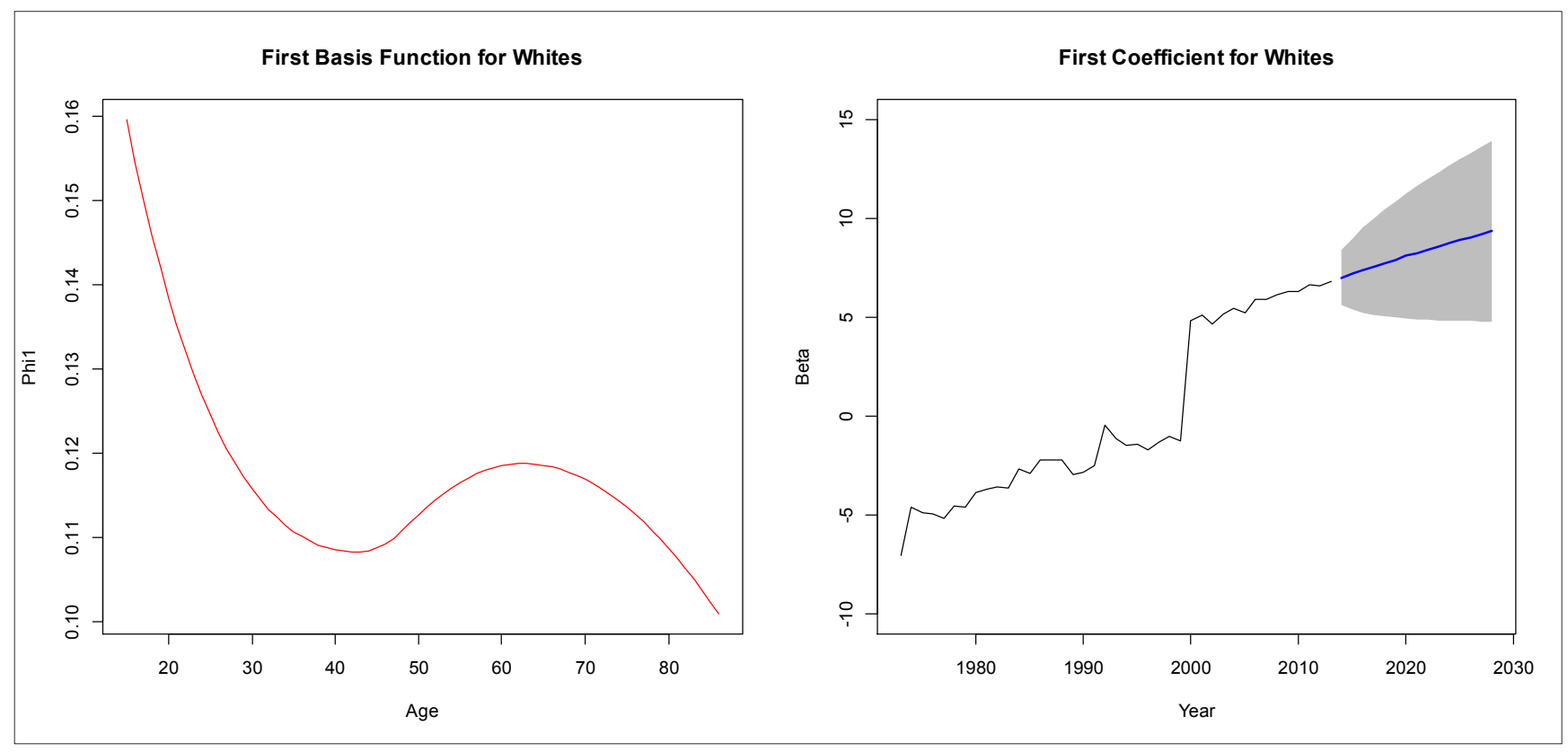

Figure 5. The first basis function and first coefficient of FTS model for breast cancer incidence data of white women.

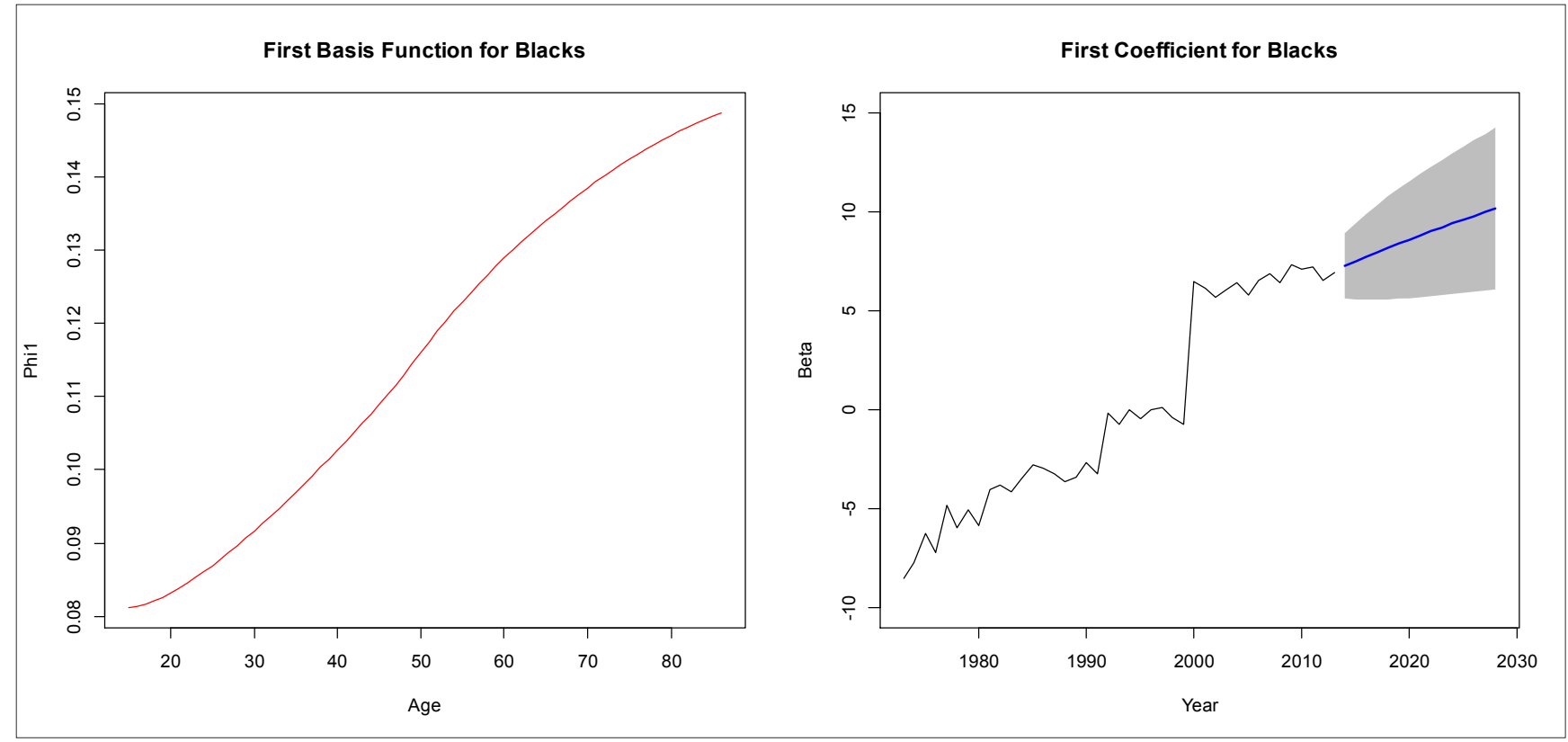

Figure 6. First basis function and first coefficient of FTS model for breast cancer incidence data of black women.

As shown in equation (3) in [9], the forecasts for the entire incidence curves are obtained by summing the results after multiplying the forecasts of each coefficient with their basis functions. These forecasts for the breast cancer incidence rates in US white and black females for next 15 years (20142028) are shown in Figure 7. This plot displays that the future incidence rates will rise for all age-groups. For whites, the expected increase in future breast cancer incidence rates is higher for older ages (55 years and above) and relatively slower in the young age-groups (under 50 years). The rates are expected to increase at highest rate among old black women (60 years and older).

The one-step forecasts (for 2014) are plotted in Figure 8; whereas 15-step forecasts (for 2028) are shown in Table 1. 
There is a little difference among whites and blacks forecasts for 2014, except a wider prediction interval for blacks of age 70 years and above. However, it can be observed from Table 1 that the future incidence rates (15-year forecasts) are expected to be relatively smaller for black women (aged 36years and under) but higher for older black women relative to the whites. The difference among the future incidence rates among the two groups is widening with age after 40 years. For the age-group 85 years, the future rates are expected to be 80.5 and 114.8 per 100,000 women in whites and blacks respectively. Similarly, the length of prediction intervals is increasing with age for both groups, and relatively broader intervals for blacks as compared to whites of the same ages.

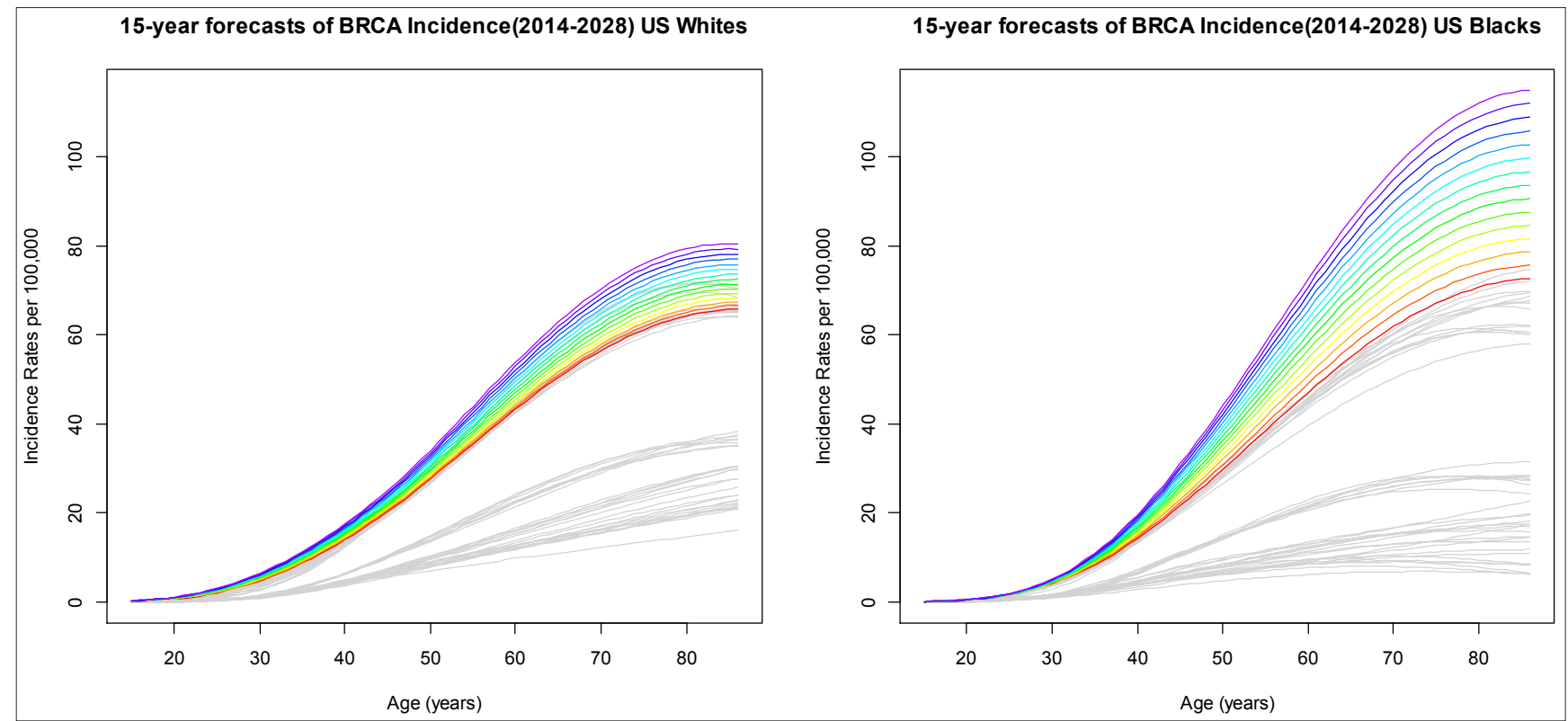

Figure 7. 15-year forecasts (2014-2028) for the Breast Cancer Incidence Rates in white and black US women. Rainbow plot [17] is used to represent the forecast curves, with earliest year (2014) are shown as red and most recent year (2028) as violet. The available data (1973-2013) are plotted as grey-shaded curves.

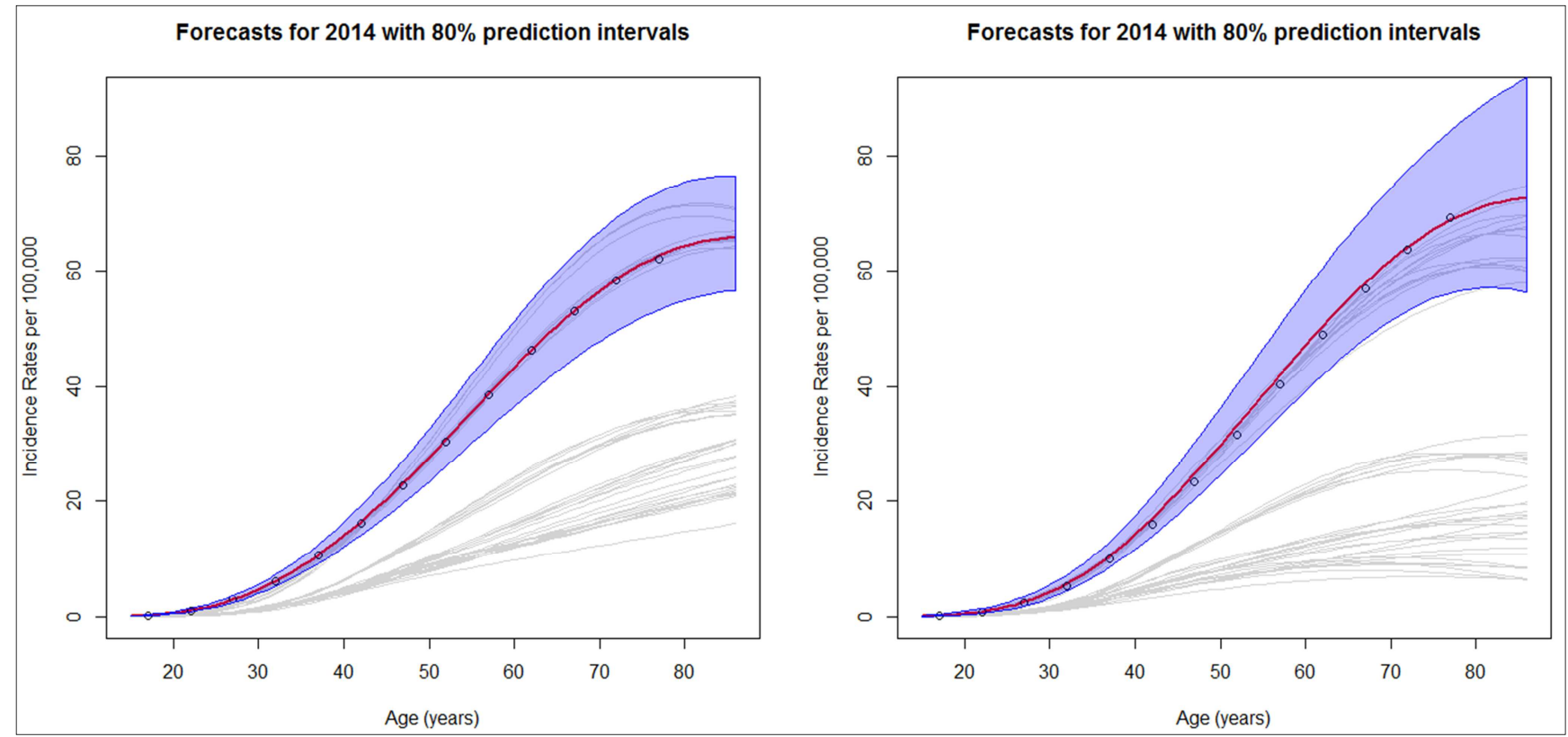

Figure 8. One-step forecasts (2014) for white and black women in US. The forecast curves are plotted with red color, whereas the data for last available year (2013) are plotted with small circles. The blue shaded regions are $80 \%$ prediction intervals. 
Table 1. 15-year forecasts (for 2028) for white and black women in US along with 80\% prediction intervals (L is the lower limit and U is the upper limit of prediction interval).

\begin{tabular}{|c|c|c|c|c|c|c|}
\hline & & Whites & & & Blacks & \\
\hline Age & Forecast & $\mathbf{L}$ & $\mathbf{U}$ & Forecast & $\mathbf{L}$ & $\mathbf{U}$ \\
\hline 15 & 0.29 & 0.04 & 1.86 & 0.09 & 0.004 & 1.76 \\
\hline 16 & 0.4 & 0.07 & 2.19 & 0.13 & 0.01 & 2.05 \\
\hline 17 & 0.53 & 0.11 & 2.55 & 0.19 & 0.16 & 2.38 \\
\hline 18 & 0.7 & 0.16 & 2.95 & 0.28 & 0.03 & 2.75 \\
\hline 19 & 0.9 & 0.24 & 3.37 & 0.38 & 0.04 & 3.15 \\
\hline 20 & 1.14 & 0.34 & 3.38 & 0.52 & 0.08 & 3.58 \\
\hline 21 & 1.43 & 0.47 & 4.33 & 0.7 & 0.12 & 4.05 \\
\hline 22 & 1.76 & 0.64 & 4.87 & 0.92 & 0.18 & 4.57 \\
\hline 23 & 2.15 & 0.85 & 5.44 & 1.2 & 0.28 & 5.12 \\
\hline 24 & 2.6 & 1.11 & 6.05 & 1.53 & 0.41 & 5.71 \\
\hline 25 & 3.09 & 1.42 & 6.71 & 1.93 & 0.58 & 6.34 \\
\hline 26 & 3.64 & 1.78 & 7.42 & 2.4 & 0.82 & 7.03 \\
\hline 27 & 4.25 & 2.21 & 8.18 & 2.95 & 1.12 & 7.75 \\
\hline 28 & 4.93 & 2.7 & 9 & 3.6 & 1.51 & 8.54 \\
\hline 29 & 5.66 & 3.23 & 9.93 & 4.32 & 1.99 & 9.4 \\
\hline 30 & 6.45 & 3.81 & 10.9 & 5.15 & 2.58 & 10.32 \\
\hline 31 & 7.31 & 4.45 & 12 & 6.09 & 3.27 & 11.37 \\
\hline 32 & 8.22 & 5.11 & 13.22 & 7.14 & 4.06 & 12.56 \\
\hline 33 & 9.2 & 5.81 & 14.56 & 8.3 & 4.94 & 13.92 \\
\hline 34 & 10.23 & 6.53 & 16.03 & 9.57 & 5.91 & 15.5 \\
\hline 35 & 11.32 & 7.26 & 17.65 & 10.96 & 6.94 & 17.32 \\
\hline 36 & 12.46 & 8.00 & 19.42 & 12.47 & 8.02 & 19.4 \\
\hline 37 & 13.66 & 8.74 & 21.35 & 14.10 & 9.14 & 21.75 \\
\hline 38 & 14.91 & 9.49 & 23.44 & 15.84 & 10.3 & 24.4 \\
\hline 39 & 16.22 & 10.25 & 25.68 & 17.7 & 11.5 & 27.27 \\
\hline 40 & 17.6 & 11 & 28.08 & 19.7 & 12.72 & 30.45 \\
\hline 41 & 18.98 & 11.76 & 30.63 & 21.76 & 14 & 33.81 \\
\hline 42 & 20.44 & 12.54 & 33.25 & 23.94 & 15.32 & 37.41 \\
\hline 43 & 21.9 & 13.32 & 36.15 & 26.22 & 16.68 & 41.22 \\
\hline 44 & 23.45 & 14.05 & 39.08 & 28.57 & 18.06 & 45.19 \\
\hline 45 & 25.01 & 14.83 & 42.17 & 30.99 & 17.49 & 49.3 \\
\hline 46 & 26.65 & 15.63 & 45.41 & 33.5 & 20.96 & 53.51 \\
\hline 47 & 28.36 & 16.48 & 48.83 & 36.06 & 22.5 & 57.81 \\
\hline 48 & 30.17 & 17.4 & 52.36 & 38.7 & 24.13 & 62.02 \\
\hline 49 & 32.05 & 18.35 & 55.99 & 41.36 & 25.85 & 66.2 \\
\hline 50 & 33.98 & 19.34 & 59.7 & 44.09 & 27.61 & 70.43 \\
\hline 51 & 35.94 & 20.36 & 63.46 & 46.88 & 29.41 & 74.72 \\
\hline 52 & 37.93 & 21.39 & 67.26 & 49.7 & 31.25 & 79.04 \\
\hline 53 & 39.93 & 22.43 & 71.09 & 52.55 & 33.12 & 83.39 \\
\hline 54 & 41.95 & 23.48 & 74.93 & 58.31 & 35.01 & 87.75 \\
\hline 55 & 43.96 & 24.54 & 78.76 & 58.31 & 36.92 & 92.1 \\
\hline 56 & 45.94 & 25.6 & 82.55 & 61.2 & 38.84 & 96.43 \\
\hline 57 & 26.67 & 86.3 & 64.08 & 40.77 & 40.77 & 100.72 \\
\hline 58 & 49.95 & 27.73 & 89.98 & 66.95 & 42.7 & 104.96 \\
\hline 59 & 51.91 & 28.79 & 93.59 & 69.79 & 42.7 & 104.96 \\
\hline 60 & 53.83 & 29.84 & 97.1 & 72.6 & 46.54 & 113.25 \\
\hline 61 & 55.72 & 30.89 & 100.5 & 75.37 & 48.43 & 117.28 \\
\hline 62 & 57.57 & 31.94 & 103.78 & 78.09 & 50.3 & 121.22 \\
\hline 63 & 59.38 & 32.97 & 106.93 & 80.75 & 52.13 & 125.08 \\
\hline 64 & 61.13 & 33.99 & 109.93 & 83.35 & 53.92 & 128.84 \\
\hline 65 & 62.83 & 35 & 112.78 & 85.88 & 55.66 & 132.51 \\
\hline 66 & 64.47 & 36 & 115.46 & 88.34 & 57.34 & 136.1 \\
\hline 67 & 66.05 & 36.98 & 117.95 & 90.71 & 58.95 & 139.6 \\
\hline 68 & 67.56 & 37.95 & 120.3 & 93 & 60.47 & 143.02 \\
\hline 69 & 69 & 38.89 & 122.44 & 95.19 & 61.91 & 146.36 \\
\hline 70 & 70.37 & 39.81 & 124.4 & 97.28 & 63.25 & 149.63 \\
\hline 71 & 71.66 & 40.71 & 126.15 & 99.28 & 64.48 & 152.83 \\
\hline 72 & 72.87 & 41.58 & 127.71 & 101.16 & 65.61 & 155.97 \\
\hline 73 & 73.99 & 42.42 & 129.07 & 102.94 & 66.61 & 159.06 \\
\hline 74 & 75.04 & 43.23 & 130.23 & 104.6 & 67.5 & 162.1 \\
\hline 75 & 75.99 & 44 & 131.19 & 106.14 & 68.24 & 165.09 \\
\hline 76 & 76.85 & 44.76 & 131.95 & 107.456 & 68.85 & 168.04 \\
\hline 77 & 77.62 & 45.47 & 132.5 & 108.87 & 69.33 & 170.95 \\
\hline 78 & 78.3 & 46.14 & 132.89 & 110.05 & 69.68 & 173.83 \\
\hline
\end{tabular}




\begin{tabular}{|c|c|c|c|c|c|c|}
\hline & & Whites & & & Blacks & \\
\hline Age & Forecast & $\mathbf{L}$ & $\mathbf{U}$ & Forecast & $\mathbf{L}$ & $\mathbf{U}$ \\
\hline 79 & 78.89 & 46.77 & 133 & 111.11 & 69.88 & 176.66 \\
\hline 80 & 79.39 & 47.6 & 132.07 & 112.05 & 69.21 & 179.45 \\
\hline 81 & 79.79 & 47.91 & 132.89 & 112.86 & 69.9 & 182.21 \\
\hline 82 & 80.1 & 48.41 & 132.54 & 113.55 & 69.72 & 184.92 \\
\hline 83 & 80.32 & 48.87 & 132.02 & 114.16 & 69.41 & 187.6 \\
\hline 84 & 80.45 & 49.27 & 131.34 & 114.55 & 68.99 & 190.2 \\
\hline 85 & 80.48 & 49.63 & 130.52 & 114.88 & 68.46 & 192.7 \\
\hline
\end{tabular}

\section{Discussion}

In the United States, for all cancer sites combined, the incidence and mortality rates in recent years have shown either measurable stabilization in observed rates, or decline [18]. However, it is observed that the race or ethnic disparities still persist in cancer incidence, mortality, and survival [19-25]. It is found previously that for all cancer sites combined, the black women have lower incidence rates but higher mortality rate than white women [20, 25]. In addition to this, for most cancer sites, black women compared with white women are more likely to have poorer stage distribution of cancer and to have lower survival rates $[18,26]$. Furthermore, it is also documented that black women have benefited less than white women from advances made in early detection and treatments [27]. Such race/ethnic disparities in cancer epidemiology are most common and evident in breast cancer incidence rates as well.

Few studies in the past considered age related changes in breast cancer incidence rates. Breast cancer tends to be diagnosed at a younger age than other common cancers. The median age at diagnosis of breast cancer is found to be 61 years, compared with 70 years for lung cancer and 68 years for colorectal cancer. [26] observed that about $19 \%$ of breast cancers are diagnosed in women ages 30-49 years, and 44\% occur among women who are age 65 years or older. In the United States, for the period 1988-2002, age-adjusted breast cancer incidence and mortality rates for white women were 134.4 and 26.4 per 100,000 women, respectively, and those for black women were 119.4 per 100,000 and 34.7 per 100,000 women [19]. Compared with black women age 50 years and above, who have had stable incidence rates since 1993, white women in the same age group have had an increase in incidence since 1987[21]. Among women age less than 50 , breast cancer incidence rates have been stable for white women since 1986 and have declined among black women since 1991[21]. Although overall age-adjusted breast cancer incidence rates were higher among white than black women and black women of age 35 years or less have higher incidence rates than white women [21].

Moreover, the survival disparities between white and black women of US persist even after adjusting for insurance status and access to health care [26]. It is observed that compared with white women, black women have $50 \%$ great risk of breast cancer-related mortality after adjusting for stage, estrogen(ER)/progesterone (PR) receptor status, surgical treatment, radiation therapy and age at diagnosis . Some of the studies suggested a conversion among the breast cancer rates of whites and blacks [27].

Although many risk factors may increase the chance of having breast cancer, it is not yet known just how some of these risk factors cause cells to become cancerous [28, 30]. Risk factors can be divided into un-modifiable risk factors and risk factors related to lifestyle choices. Un-modifiable risk factors are gender, age, genetic factors, family history, personal history of breast cancer, dense breast tissue, menstrual periods, and breast radiation early in life. Risk factors related to lifestyle are not having pregnancy history or pregnancy at late ages, recent use of birth control pills, using hormone therapy after menopause, not breast-feeding, alcohol, being overweight or obese, lack of exercise, and induced abortion are suggested by some researchers [29-33].

The risk of developing breast cancer increases as a woman ages and it is found that a woman who experiences menopause after the age 55 has an increased risk of breast, ovarian and uterine cancers. The risk is greater if a woman also began menstruating before the age of 12 years. A longer exposure to estrogen increases a woman's risk of breast cancers, and hence; a women who have been through natural menopause are more likely to develop breast cancer as twice as high due to hormonal factors [20].

Black women are still more likely to be diagnosed with later-stage disease than other women and have the lowest survival rates in each stage of diagnosis. The researchers said reasons for this are lack of regular screening; lack of followup after suspicious screening results; lack of access to timely, high-quality treatment; and a higher percentage of aggressive, harder-to-treat tumors. Most experts think that it's likely to be a combination of factors. Rising obesity rates among black women, coupled with statistics showing that more black women are having fewer children and having children later in life could play a role. We also know that black women are less likely to participate in clinical trials testing new breast cancer treatments. Some doctors think that it's possible that some medicines work differently or less effectively in black women.

Women of all ethnicities (including both whites and blacks) can take steps to keep their risk of breast cancer as low as it can be. Late stage at presentation of breast cancer is the main problem and possesses a challenge to the health care community. For black woman, it was recommended in [34] to talk to their doctor about their risk of breast cancer, as well as about lifestyle choices they could make to lower that risk. It includes maintaining a healthy weight, eating a healthy diet that is low in processed foods, sugar, and trans fats, 
exercising every day and limiting/avoiding alcohol and smoking.

\section{Conclusion}

In this paper, we applied functional time series (FTS) models to the age-specific breast cancer incidence rates of whites and black women in the United Stated. By plotting the age-specific incidence rates, we observed that the breast cancer incidence rates increased with age for both groups. From early ages, the overall incidence rates showed a clear increasing trend through age of 50. In most recent years, the rates declined for the white women of age $50+$, whereas they increased for the black women of age 50 years and older.

The forecast plot depicts that the future incidence rates are expected to rise for all age-groups. For whites, the expected increase in future breast cancer incidence rates is higher for older ages ( 55 years and above) and relatively slower in the young age-groups (under 50 years). However, the future incidence rates are expected to increase at a higher rate among old black women (60 years and older). On the basis of our analysis, the black/white disparity among the breast cancer incidence rates is expected to be widening in future.

Finally, the findings of this study support the need for innovative research, especially on the differential epidemiology of breast cancer. There is also a need for equal delivery of quality care to eliminate breast cancer disparities among white and black women in the United States.

\section{References}

[1] Ferlay J, Shin HR, Bray F, Forman D, Mathers C, Parkin DM, Estimates of worldwide burden of cancer in 2008: GLOBOCAN 2008, Int J Cancer. 127 (2010) 2893-917.

[2] Cancer Treatment And Survivorship Statistics, 2016 CA: A Cancer Journal For Clinicians (2016) https://www.researchgate.net/publication/303769019.

[3] Li CI, Malone KE, Daling JR. Differences in breast cancer stage, treatment, and survival by race and ethnicity, Arch Intern Med. 163(1) (2003) 49-56.

[4] Chu KC, Lamar CA, Freeman HP. Racial disparities in breast carcinoma survival rates: separating factors that affect diagnosis from factors that affect treatment, Cancer, 97(11) (2003) 2853-2860.

[5] Li CI, Malone KE, Daling JR. Differences in breast cancer hormone receptor status and histology by race and ethnicity among women 50 years of age and older, Cancer Epidemiology Biomarkers Prev.11(7) (2002) 601-607.

[6] Hyndman R.J. and Ullah, M. S, Robust forecasting of mortality and fertility rates: A functional data approach. Computational Statistics \& Data Analysis, 51 (2007) 4942 4956.

[7] Erbas, B., Hyndman, R. J. and Gertig, D.M., Forecasting agespecific breast cancer mortality using functional data models. Statistics in Medicine, 26 (2007) 458-470.

[8] Yasmeen, F., Hyndman, R. J. and Erbas, B., Forecasting age- related changes in breast cancer mortality among white and black US women: A functional data approach. Cancer Epidemiology, 34(5) (2010) 542-549.

[9] Yasmeen, F., Coherent Forecasts of US Age-specific Breast Cancer Mortality, Donnish Journal of Cancer Research and Experimental Oncology, 1(1) (2015)001-008, http:// www.donnishjournals.org/djcreo.

[10] Yasmeen, F., Zaheer, S., Functional time series models to estimate future age specific breast cancer incidence rates for women in Karachi, Pakistan. J Health Sci,2(2014)213-221.

[11] Zaheer, S. and Yasmeen, F., Breast Cancer Incidence Rates in Karachi, Proceeding of $10^{\text {th }}$ International Conference on Statistical Sciences (ISOSS), March 7-9, Lahore, Pakistan, 24 (2013) 71-78.

[12] Yasmeen, F., Modeling Breast Cancer Incidence Rates: A Comparison between the Components of Functional Time Series (FTS) Model applied on Karachi (Pakistan) and US Data, Open Journal of Applied Sciences, 6(2016) 524-533.

[13] Yasmeen, F. and Mughal, S .Functional Time Series Models and the APC Models: A Comparative Study on the Lung Cancer Incidence Rates in Denmark, Journal of US-China Medical Science, ISSN 1548-6648, USA, 11(3)(2014) 121128.

[14] Surveillance, Epidemiology, and End Results (SEER) Program. Delay-Adjusted Incidence database: "SEER Incidence Delay- Adjusted Rates, 9 Registries, 1975-2013". National Cancer Institute, DCCPS, Surveillance Research Program, Statistical Research and Applications Branch, released April based on the November 2015 SEER data submission.

[15] Hyndman, R. J., Demography: Forecasting mortality, fertility, migration and population data, R. package, version 1.18, with contribution from Heather Booth, Leonie Tickle and John Maindonald(2014)(http://robjhyndman.com/software/demogra phy/).

[16] Hyndman, R.J., Forecast: Forecasting functions of time series models, R. package, Version 7.1(2014) (http://robjhyndman.com/software/forecast/).

[17] Hyndman R J and Shang H.L. Rainbow plots, bagplots and boxplots for functional data. J Comput Graph Stat, 19(1) (2010)29-45.

[18] Jemal A, Clegg LX, Ward E, et al. Annual report to the nation on the status of cancer, 1975-2001, with a special feature regarding survival. Cancer. 101(1)(2004) 3-27.

[19] Jemal A, Siegel R, Ward E. Cancer statistics, 2006. CA Cancer J Clin. 56(2) (2006) 106-130.

[20] US Department of Health and Human Services. National Healthcare Disparities Report. US Department of Health and Human Services, Agency for Health Care Research and Quality; Rockville, MD: (2003).

[21] Smigal C, Jemal A, Ward E, et al. Trends in breast cancer by race and ethnicity: update 2006. CA Cancer J Clin.56(3) (2006) 168-183.

[22] Tammemagi CM, Nerenz D, Neslund-Dudas C, Feldkamp C, Nathanson D. Comorbidity and survival disparities among black and white patients with breast cancer. JAMA.294(14) (2005) 1765-1772. 
[23] Newman LA. Breast cancer in African-American women. Oncologist 10(1) (2005) 1-14.

[24] Newman LA, Griffith KA, Jatoi I, Simon MS, Crowe JP, Colditz GA. Meta-analysis of survival in African American and white American patients with breast cancer: ethnicity compared with socioeconomic status. J ClinOncol. 24(9) (2006) 1342-1349.

[25] Chlebowski RT, Chen Z, Anderson GL. Ethnicity and breast cancer: factors influencing differences in incidence and outcome. J Natl Cancer Inst. 97(6) (2005) 439-448.

[26] Field TS, Buist DS, Doubeni C. Disparities and survival among breast cancer patients. J Natl Cancer InstMonogr 35(2005) 88-95.

[27] DeSantis CE, Fedewa SA, Goding Sauer A, Kramer JL, Smith RA, Jemal A. Breast cancer statistics, 2015: Convergence of incidence rates between black and white women. CA Cancer J Clin., 66(1) (2016) 31-42.
[28] Chu KC, Tarone RE, Brawley OW. Breast cancer trends of black women compared with white women. Arch Fam Med. 8(6) (1999) 521-528.

[29] Cancer treatment and survivorship statistics, 2016, CA A Cancer Journal for Clinicians, (2016) https://www.researchgate.net/publication/303769019.

[30] Atlanta: American Cancer Society; 2013. American Cancer Society. Breast Cancer Facts and Figures (2013).

[31] Cooper K. Springhouse: Springhouse Corp, Pathophysiology Made Incredibly Easy. (1998).

[32] Gallucci BB. Selected concepts of cancer as a disease: From the Greeks to 1900. OncolNurs Forum.12 (1985) 67-71.

[33] Atlanta: American Cancer Society; 2009. American Cancer Society. Recommendations for the Early Detection of Cancer.

[34] Edge S, Byrd DR, Compton CC, Fritz AG, Greene FL, Trotti A, editors. 7th ed. New York: Springer AJCC Cancer Staging Manual (2010)347-69. 